\title{
Correction: The american pediatric society and society for pediatric research joint statement against racism and social injustice
}

Steven H. Abman (D), Sarah Armstrong, Susan Baker, Clifford W. Bogue, Waldemar Carlo, Lina Chalak, Stephen R. Daniels, Stephanie Davis, Michael R. Debaun, Candice Fike, Lauren Frazer, Keisha Gibson, Michelle Gill, Hannah Glass, Catherine M. Gordon, Monika Goyal, Joel Hirschhorn, Lori Holtz, David A. Hunstad, Mary B. Leonard, Nathalie Maitre, Larry Markham, Linda McAllister-Lucas, Jordan Orange, Prachi Shah, Tamara Simon, Robin H. Steinhorn, Beth Tarini and Leslie R. Walker-Harding

(c) The Author(s), under exclusive licence to the International Pediatric Research Foundation, Inc 2021

Pediatric Research (2022) 91:264; https://doi.org/10.1038/s41390-021-01677-2

Correction to: Pediatric Research https://doi.org/10.1038/s41390-

020-01107-9, published online 3 September 2020
In this article, the names of the authors David A. Hunstad and Tamara Simon have been updated. The original article has been corrected. 